\title{
Targeted and restricted complement activation on acrosome-reacted spermatozoa
}

\author{
Rebecca C. Riley-Vargas, ${ }^{1}$ Susan Lanzendorf, ${ }^{2}$ and John P. Atkinson ${ }^{1}$ \\ 1Department of Medicine, Rheumatology Division, and 2Department of Obstetrics and Gynecology, \\ Washington University School of Medicine, St. Louis, Missouri, USA.
}

\begin{abstract}
A specific hypoglycosylated isoform of the complement regulator membrane cofactor protein (MCP; CD46) is expressed on the inner acrosomal membrane (IAM) of spermatozoa. This membrane is exposed after the acrosome reaction, an exocytosis event that occurs upon contact with the zona pellucida. We initiated this investigation to assess MCP's regulatory function in situ on spermatozoa. Upon exposure of human spermatozoa to autologous serum or follicular fluid, we unexpectedly observed that acrosome-reacted spermatozoa activated the complement cascade efficiently through $\mathrm{C} 3$ but not beyond. Using FACS to simultaneously evaluate viability, acrosomal status, and complement deposition, we found that complement activation was initiated by $\mathrm{C}$-reactive protein (CRP) and was $\mathrm{C} 1 \mathrm{q}, \mathrm{C} 2$, and factor $\mathrm{B}$ dependent. This pattern is consistent with engagement of the classical pathway followed by amplification through the alternative pathway. C3b deposition was targeted to the IAM, where it was cleaved to C3bi. Factor $\mathrm{H}$, and not MCP, was the cofactor responsible for $\mathrm{C} 3 \mathrm{~b}$ cleavage. We propose that this localized deposition of complement fragments aids in the fusion process between the spermatozoa and egg, in a role akin to that of complement in immune adherence. In addition, we speculate that this "targeted and restricted" form of complement activation on host cells is a common strategy to handle modified self.
\end{abstract}

\section{Introduction}

Membrane cofactor protein (MCP; CD46) is a widely expressed complement regulator with cofactor activity for the factor Imediated cleavage of $\mathrm{C} 3 \mathrm{~b}$ and $\mathrm{C} 4 \mathrm{~b}(1-4)$. In addition, MCP is a receptor for a growing list of human pathogens, including 4 viruses $(5-10)$ and 3 bacteria $(11,12)$. Cross-linking MCP produces intracellular signals in diverse cell types including macrophages (reviewed in ref. 13), $\mathrm{CD}^{+} \mathrm{T}$ cells (14), and epithelial cells (reviewed in ref. 13). Recently, a deficiency of MCP has been linked to the hemolytic uremic syndrome $(15,16)$.

MCP plays a role in reproduction (reviewed in ref. 13). The MCP isoform expressed on spermatozoa is independent of the inherited size polymorphism observed in somatic cells (17) and is hypoglycosylated secondary to trimming of the mature N-linked sugars (18). Its binding affinity and cofactor activity for $\mathrm{C} 3 \mathrm{~b}$ and $\mathrm{C} 4 \mathrm{~b}$, however, are comparable to those of MCP expressed by somatic cells (2). Expression of MCP by human spermatozoa is restricted to the inner acrosomal membrane (IAM) $(19,20)$, which is exposed following binding of spermatozoa to the zona pellucida (Figure 1). Variations in MCP expression on spermatozoa have been associated with infertility $(21,22)$, and MCP is a target for anti-sperm Abs (23). In mice (24), rats (25), and probably guinea pigs (26), MCP is expressed exclusively on the IAM of spermatozoa. In these species, another related protein, Crry, is widely expressed and performs MCP's complement-regulatory role $(27,28)$. Also, Abs against MCP inhibit binding to and penetration of zona-free hamster eggs $(19,29,30)$

Nonstandard abbreviations used: AR, acrosome-reacted; CCP1, complement control protein repeat 1; CRP, C-reactive protein; DAF, decay-accelerating factor; $\mathrm{GVB}^{++}$, gelatin veronal buffer supplemented with $\mathrm{Mg}^{2+}$ and $\mathrm{Ca}^{2+}$; IAM, inner acrosomal membrane; MCP, membrane cofactor protein; $\mathrm{mHTF}$, modified human tubal fluid; TRACS, targeted and restricted activation of the complement system.

Conflict of interest: The authors have declared that no conflict of interest exists.

Citation for this article: J. Clin. Invest. 115:1241-1249 (2005).

doi:10.1172/JCI200523213 and human zona (31) by human spermatozoa. In particular, Abs against complement control protein repeat 1 (CCP1), the aminoterminal CCP, block binding more efficiently than function-blocking Abs that bind to CCPs 3 and 4 (30). This observation led us to evaluate MCP expression on spermatozoa of New World monkeys (32), since they express a splice alternative of MCP lacking CCP1 on somatic cells (33). This variant may protect against infection by measles virus, whose hemagglutinin requires CCP1 to bind MCP (33). However, the loss of this repeat does not affect MCP's complement-regulatory ability (33-35). Surprisingly, New World monkeys retain expression of CCP1 on spermatozoa (32). This conservation of structure suggests that MCP participates in fertilization, in addition to being a complement regulator.

There are several explanations for MCP's expression on the IAM. First, as noted, the retention of CCP1 on spermatozoa of New World monkeys and the Ab inhibition data both suggest that CCP1 might be playing a role in fertilization independent of complement activation (32). Second, in 1993 Anderson et al. described a model whereby MCP might be one half of a C3b dimer bridge between the spermatozoa and the egg (29). The expression of complement receptors 1 and 3 on the plasma membrane of human eggs adds support to this possibility (29). Third, the signaling capabilities of MCP, especially the ability to induce a calcium flux (reviewed in ref. 13), may be important on the IAM. Fourth, MCP's complement-regulatory activity could protect acrosome-reacted (AR) spermatozoa during the final steps of fertilization. Relatedly, the concentration of complement components in follicular fluid is equivalent to that in serum (36-39), and acrosin, a protease released during the acrosome reaction, can cleave C3 (29). However, with anti-sperm Abs and other potential complement-activating agents in follicular fluid, it is curious that MCP is not also expressed on the plasma membrane of spermatozoa as are 2 other complement-regulatory proteins, decay-accelerating factor (DAF) (40) and CD59 (41). 


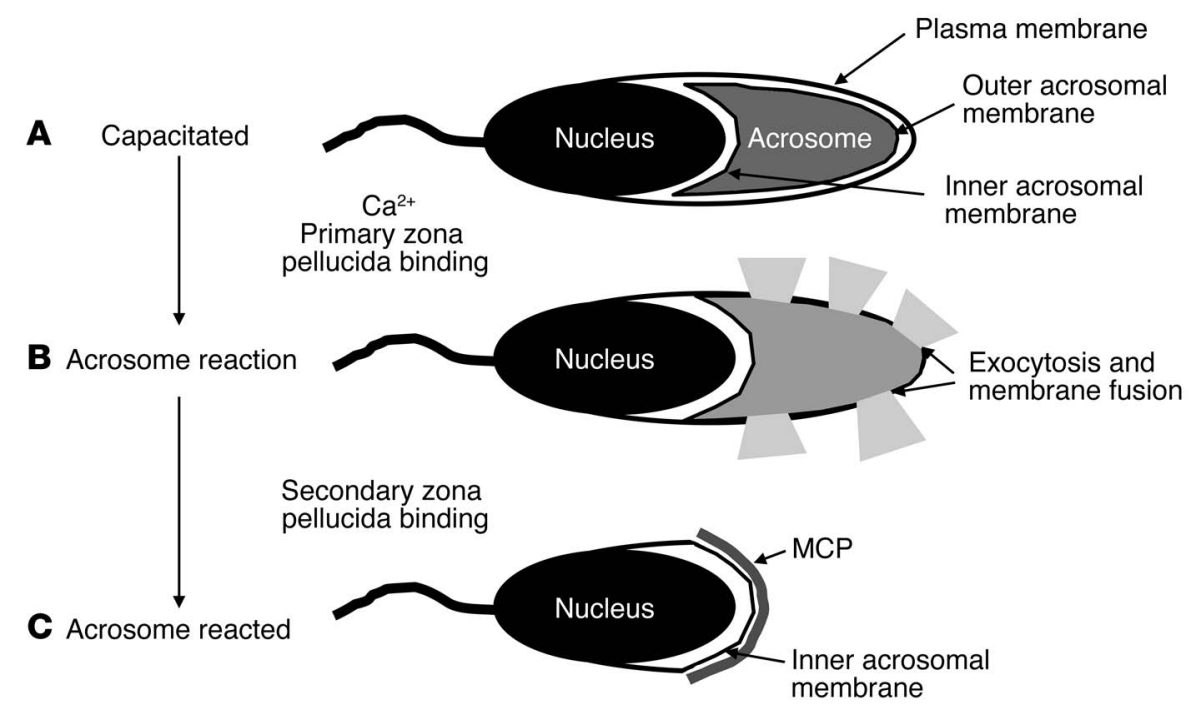

Figure 1

Schematic representation of the acrosome reaction of human spermatozoa. (A) The acrosome reaction of capacitated spermatozoa occurs after binding to the zona pellucida. (B) Upon this event, the outer acrosomal membrane fuses with the plasma membrane, releasing the acrosome contents. (C) MCP is expressed on the IAM and thus is exposed following the acrosome reaction.

To better define the role of MCP on spermatozoa, we set out to evaluate MCP in situ as a complement regulator on spermatozoa. Early in the course of these experiments, we recognized that AR human spermatozoa spontaneously activate complement, and this report represents our initial studies of this phenomenon.

\section{Results}

The IAM of spermatozoa is exposed following the acrosome reaction. Since MCP's expression is limited to this structure, the reaction of anti-MCP specific mAb TRA-2-10 with spermatozoa provides a means to define acrosomal status (i.e., AR or nonreacted). Spermatozoa that do not stain for MCP have an intact plasma membrane and therefore are not AR. MCP-positive spermatozoa, as determined by FACS, have an exposed IAM because they either are $\mathrm{AR}$ or have a compromised plasma membrane. The latter possibility was assessed using membrane-impermeable dyes to mark nonviable (i.e., membrane-compromised) spermatozoa. Consistent with the literature, we found that approximately $25 \%$ of the spermatozoa in fresh ejaculates were in this category.

To induce the acrosome reaction, human spermatozoa were incubated with the calcium ionophore A23187. This reagent converts between $30 \%$ and $70 \%$ of viable, nonreacted spermatozoa to AR spermatozoa. A representative histogram of MCP expression by ionophore-treated spermatozoa is shown in Figure 2A. Using primary labeled Abs, there is approximately a 1-log shift in MCP expression following incubation of spermatozoa with the ionophore. This shift represents exposure of the IAM and hence MCP. In this sample, approximately $60 \%$ of the spermatozoa were induced to acrosome-react. A small percentage of cells (approximately $20 \%$ for this donor) in the untreated sample were also MCP positive. This is expected and represents the population of spermatozoa that have spontaneously undergone the acrosome reaction. The proportion of spontaneously AR spermatozoa varied among donors and ranged from $20 \%$ to $45 \%$ in the 4 donors regularly used for these studies.

To examine the complement-regulatory activity of MCP on spermatozoa in situ, experiments were designed in which spermatozoa were to be sensitized with anti-sperm Abs. As a control, unsensitized ionophore-treated and untreated spermatozoa were exposed to $10 \%$ autologous serum and analyzed by FACS. An
$\mathrm{mAb}$ against $\mathrm{C} 3 \mathrm{~d}$ was used as a marker of complement activation. Unexpectedly, this $\mathrm{mAb}$ reacted with unsensitized, ionophoretreated spermatozoa. This suggests that, following the acrosome reaction, spermatozoa activate complement (Figure 2B). To further analyze this finding, we stained for MCP and C3d on the same population. There was a direct relationship between MCP staining and presence of C3d antigen (Figure 3A). No staining for C3d was observed on viable nonreacted spermatozoa (Figure 3A, box in top left panel). This result further establishes that C3-derived fragments deposit on AR spermatozoa. Although there was variability in the percentage of AR spermatozoa among individuals, the parallel relationship between MCP and C3d staining was observed for each donor (Figure 3A), and the pattern was reproducible for the same donor. The deposition on MCP-positive (AR) spermatozoa was greater (mean \pm SD fluorescence for 4 donors was $313 \pm 65$ ) than on spermatozoa incubated with heat-inactivated serum (mean \pm SD fluorescence for 4 donors
A

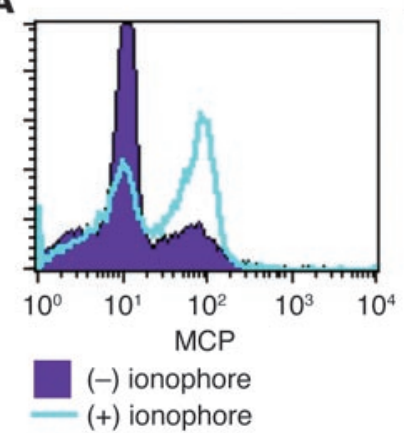

B

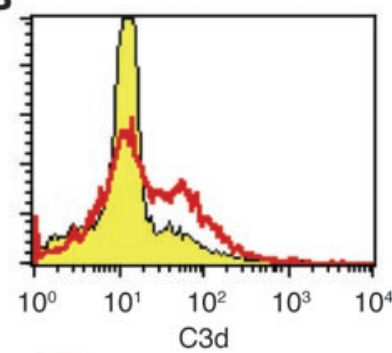

$(-)$ ionophore

(+) ionophore

\section{Figure 2}

FACS profiles of MCP and C3d on ionophore-treated spermatozoa. (A) Calcium ionophore induces the acrosome reaction, exposing MCP to the fluorescently labeled Ab. Approximately $20 \%$ of spermatozoa spontaneously undergo the acrosome reaction in the untreated sample. The antibody used was Alexa Fluor 488-labeled TRA-2-10 (mAb against MCP). (B) Spermatozoa exposed to calcium ionophore activate complement monitored by C3d deposition. The antibody used was Alexa Fluor 647-labeled mAb against $\mathrm{C} 3 \mathrm{~d}$. A representative experiment (out of about 50 experiments) is shown. 
A

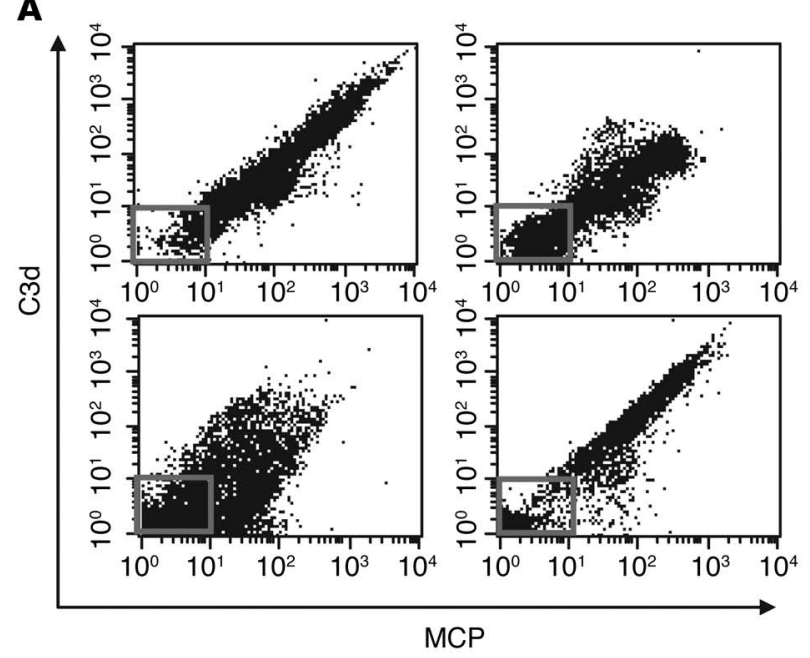

B

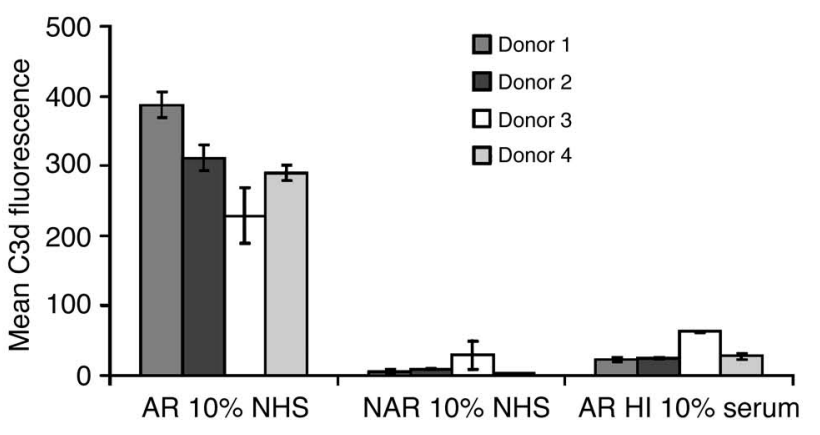

Figure 3

FACS analysis of C3d deposition on viable spermatozoa exposed to 10\% autologous serum. (A) Each FACS plot represents a single donor and is gated on viable spermatozoa. The correlation between the acrosome reaction (as measured by MCP staining) and complement deposition (as measured by C3d staining) is observed in all 4 donors. The inset box in each FACS plot represents the MCP- and C3d-negative population. (B) Mean C3d fluorescence of viable AR and non-AR (NAR) spermatozoa exposed to $10 \%$ normal human serum (NHS) or heat-inactivated (HI) human serum. Mean \pm SD for a minimum of 3 experiments per donor is shown.

was $34 \pm 17$, which is equivalent to that for the no-Ab control samples) (Figure 3B). Complement deposition was rapid, being maximal by 15 minutes, and there was no decrease in spermatozoa viability at 15 or 30 minutes. In addition, after 15 minutes only approximately $1 \%$ of the viable C3-positive spermatozoa were positive for C5b-9 neoepitope (data not shown), and there was no decrease in spermatozoa motility. Also, we were not able to detect $\mathrm{C} 5 \mathrm{~b}$ or $\mathrm{C} 9$ deposition on ionophore-treated spermatozoa by Western blot, even if the cofactor activity of MCP was blocked with an $\mathrm{mAb}$ (data not shown). These data establish that the complement deposition on AR spermatozoa is restricted to the early complement components (through C3) and does not decrease spermatozoa viability.

Following binding to the zona pellucida in the fallopian tubes, which induces the acrosome reaction, the IAM of spermatozoa is exposed to follicular fluid. We obtained neat follicular fluid from 1 donor and follicular fluid diluted approximately 1:10 from several donors to determine whether the complement deposition occurs in this physiologically relevant source of complement. We incubated both untreated and ionophore-treated spermatozoa with follicular fluid and observed deposition on AR spermatozoa (Figure 4). Results were similar whether neat or diluted follicular fluid was used. The mean $( \pm$ SD) fluores-

Figure 4

FACS analysis of viable spermatozoa exposed to follicular fluid. (A) Each FACS plot represents a single donor and is gated on viable spermatozoa. The correlation between the acrosome reaction (as measured by MCP staining) and complement deposition (as measured by C3d deposition) is observed for both donors. Each FACS plot is representative of 10 experiments. (B) Mean C3d fluorescence of viable spermatozoa exposed to follicular fluid (FF). Because of the limited quantity of neat follicular fluid, only 1 experiment using donor 1 was performed. Data for donor 3 are the mean \pm SD of 3 experiments. cence for C3d was $263 \pm 19$ and 219 (see legend to Figure 4) for spermatozoa from 2 different donors incubated with follicular fluid, compared with $387 \pm 17$ and $229 \pm 39$, respectively, for spermatozoa incubated with $10 \%$ serum.

To evaluate the pathway responsible for complement activation, deficient, depleted, and treated human sera were used (Table 1). Deposition of C3 fragments was prevented if the autologous serum was heat-inactivated or treated with EDTA. These conditions inactivate the proteases in the complement cascade but leave $\mathrm{C} 3$ intact. In these conditions there was no complement activation; this indicates that the initial activation

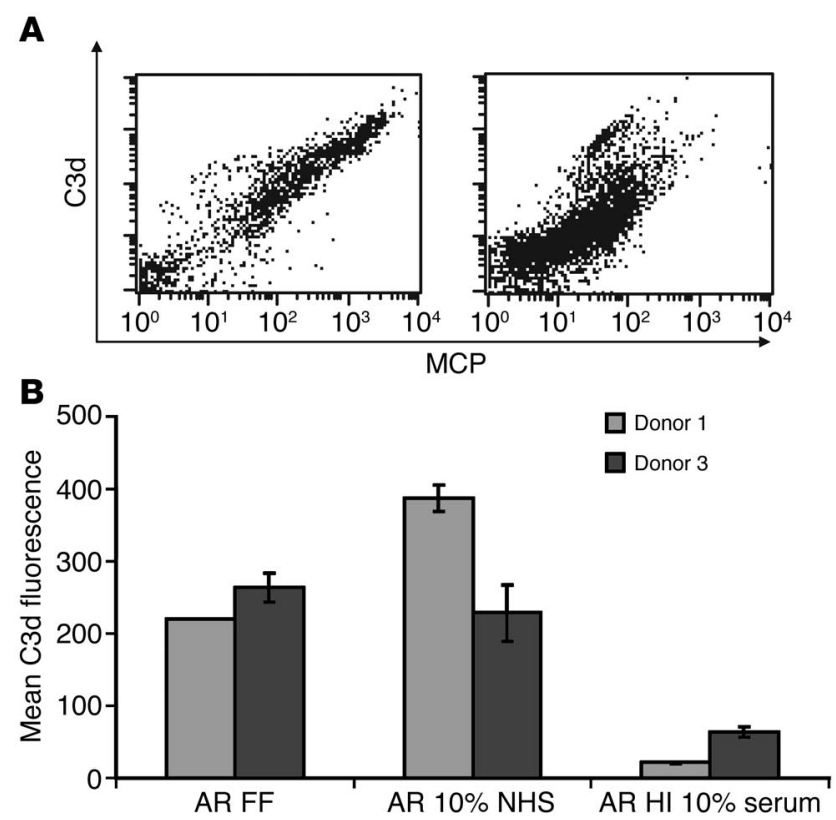




\section{Table 1}

Complement activation by AR spermatozoa under different serum conditions

\begin{tabular}{lcc} 
Serum & C3d & Fluorescence (mean \pm SD) \\
$10 \%$ NHS & Yes & $313 \pm 65$ \\
Heat-inactivated & No & $34 \pm 17$ \\
EDTA & No & $31 \pm 18$ \\
MgEGTA & No & $32 \pm 17$ \\
C1q-depleted & No & $29 \pm 14$ \\
C2-deficient & No & $33 \pm 12$ \\
Factor B-depleted & No & $32 \pm 18$ \\
C7-deficient & Yes & $289 \pm 54$ \\
\hline
\end{tabular}

Calcium ionophore-treated human spermatozoa were exposed for 15 minutes to the sera noted above and then incubated with an $\mathrm{mAb}$ to $\mathrm{C} 3 \mathrm{~d}$ and analyzed by FACS. Heat inactivation and EDTA treatment block all complement activation pathways. Mg $^{++}$EGTA-treated serum, C1q-depleted serum, and C2-deficient serum prevent classical-pathway activation. Factor B-depleted serum prevents alternative-pathway activation. The data in the first, second, and last rows represent 10 separate experiments, while the results in the other rows are from 2 experiments. NHS, normal human serum.

of C3 is unlikely to be secondary to cleavage by an acrosomal protease such as acrosin (29). Deposition was not observed with $\mathrm{C} 1 \mathrm{q}$-depleted serum; this rules out the lectin pathway as a contributing cascade and establishes a role for the classical pathway. This conclusion was substantiated by the finding that neither C2-deficient sera nor $\mathrm{Mg}^{2+}$-EGTA-treated serum permitted complement activation. Factor B-depleted sera also prevented C3 fragment deposition, demonstrating a requirement for the alternative pathway. As anticipated, a deficiency of a late complement component, in this case C7, did not affect C3 fragment deposition. Taken together, these data support initial complement activation via the classical pathway and then amplification of the activation process through the feedback loop of the alternative pathway.
There are several means to initiate complement activation through C1q. First, we examined spermatozoa, following exposure to serum, for the presence of natural Ab. FACS, ELISA, and confocal microscopy using Abs against human IgM or IgG did not detect Ab bound to AR spermatozoa (data not shown). Second, because C-reactive protein (CRP) initiates the classical pathway via C1q (42) and is present in follicular fluid at levels similar to those in serum $(43,44)$, we performed Western blots and FACS analysis on ionophore-treated spermatozoa exposed to $10 \%$ autologous serum. A CRP-specific band at $25 \mathrm{kDa}$ was observed in AR spermatozoa exposed to serum (Figure 5A). The fainter band in the untreated sample is likely derived from spontaneously AR spermatozoa (typically $10-20 \%$ of the spermatozoa). In addition, FACS showed CRP binding to AR spermatozoa that had been exposed to $10 \%$ serum (Figure 5B). Because of these results, CRP was absorbed from serum with beads linked to phosphocholine. The resulting serum had a reduced ability to activate complement on AR spermatozoa (Figure 5C). These data demonstrate that CRP is responsible for initiating the classical complement pathway on AR spermatozoa.

Next, we used confocal microscopy to define the location of the complement fragments. An mAb against MCP, TRA-2-10, was used to identify the IAM (Figure 6A). After spermatozoa were exposed to $10 \%$ serum, an $\mathrm{mAb}$ against $\mathrm{C} 3 \mathrm{~d}$ exclusively stained the IAM (Figure $6, \mathrm{~B}$ and $\mathrm{C}$ ). This demonstrates that complement activation is restricted to the IAM and supports the FACS profile of Figure 3 in which AR spermatozoa are shown to activate complement.

The $\mathrm{Ab}$ used in the FACS and confocal experiments recognizes $\mathrm{C} 3 \mathrm{~d}$, an epitope present in all membrane-bound cleavage fragments of C3 (Figure 7A). To determine the complement fragments containing C3d on the IAM, we performed Western blots with a polyclonal $\mathrm{Ab}$ against $\mathrm{C} 3$. In the lane derived from ionophore-treated spermatozoa, the $\alpha^{\prime}$ chain is cleaved and $\alpha 2$ is present, indicating that the $\mathrm{C} 3 \mathrm{~b}$ has been cleaved to C3bi (Figure $7 \mathrm{~B}$ ). As expected, the $\alpha 1$ band is barely visible, as it would be attached via an ester linkage to proteins of varying molecular weights expressed on the IAM. Since MCP is a cofactor for the
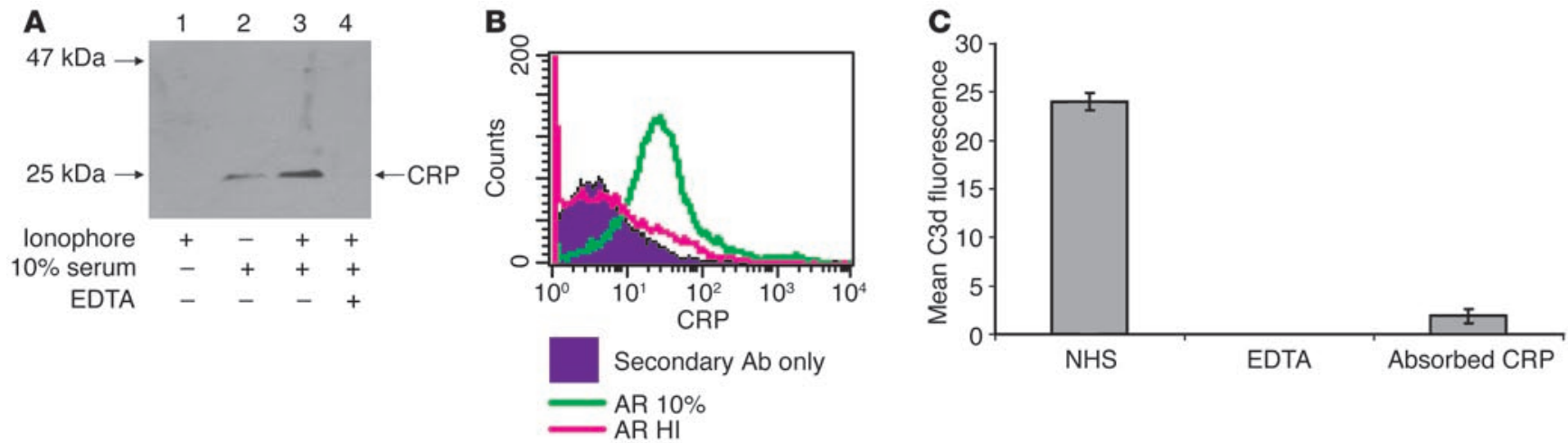

\section{Figure 5}

Analysis of CRP binding to AR spermatozoa. (A) Western blot of human spermatozoa. lonophore-treated or untreated spermatozoa were exposed to either $10 \%$ normal or EDTA-treated autologous human serum. Lysates were separated in reducing conditions by SDS-PAGE on a 10-20\% Tris-glycine gel. The primary antibody used was polyclonal goat anti-human CRP at 1:1,000 dilution. CRP is a pentamer composed of five 25-kDa subunits. (B) FACS analysis of CRP on spermatozoa. Ionophore-treated spermatozoa were exposed to either $10 \%$ normal or heat-inactivated autologous human serum. The primary antibody used was polyclonal goat anti-human CRP at 1:1,000 dilution. The secondary antibody used was FITC-linked rabbit anti-goat at 1:10,000 dilution. (C) FACS analysis of spermatozoa exposed to CRP-absorbed serum. Serum was preincubated with immobilized phosphorylcholine to absorb CRP. Spermatozoa were then exposed to 10\% normal serum, 10\% CRP-absorbed serum, or EDTA-treated human serum. Complement activation was measured by C3d fluorescence of viable AR spermatozoa. 
A

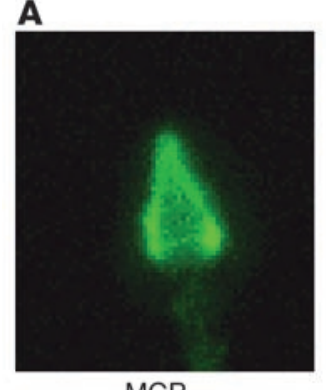

MCP

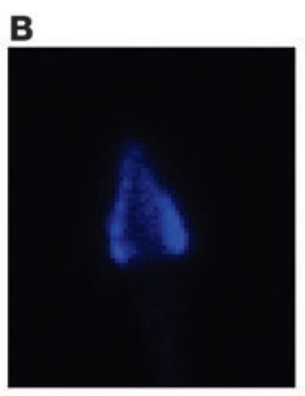

C3d

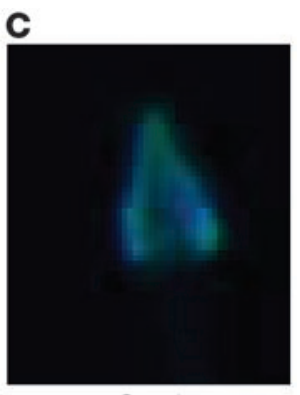

Overlay

\section{Figure 6}

Confocal microscopy of AR spermatozoa exposed to $10 \%$ serum. Spermatozoa were incubated with calcium ionophore to induce the acrosome reaction and then exposed to $10 \%$ autologous serum. (A) The mAb TRA-2-10 labeled with Alexa Fluor 488 recognizes MCP on the IAM. (B) An mAb to C3d labeled with Alexa Fluor 647 binds to the IAM. (C) Overlay of $\mathbf{A}$ and $\mathbf{B}$. C3d deposition is limited to the IAM. Magnification, $\times 63$.

cleavage of C3b to C3bi, we blocked MCP's cofactor activity with the mAb GB24 (45). TRA-2-10, used as a control, binds to CCP1 and does not block function (45). Neither Ab inhibited the cleavage of C3b to C3bi (Figure 7B). Factor H, a soluble complement regulator that is present in follicular fluid at levels comparable to those in serum (39), also cleaves C3b to C3bi. When the $\mathrm{mAb}$ $\mathrm{MH}-10$, which blocks the function of factor $\mathrm{H}$, was used (46), the cleavage of $\mathrm{C} 3 \mathrm{~b}$ was inhibited (Figure 7B). Thus, factor $\mathrm{H}$ was responsible for the majority of the $\mathrm{C} 3 \mathrm{~b}$ cleavage.

\section{Discussion}

The acrosome reaction is a large exocytosis event that is induced in vivo when spermatozoa bind to the zona pellucida of the egg (Figure 1). This reaction releases enzymes that degrade the protein matrix of the zona and thereby allows the spermatozoa to transverse the zona and enter the perivitelline space. Our data show that when this event occurs spontaneously or is induced in vitro, complement activation is triggered, resulting in deposition of complement fragments specifically on the IAM. Deposition was dependent on $\mathrm{C} 1 \mathrm{q}, \mathrm{C} 2$, and factor $\mathrm{B}$, consistent with initial activation of the classical pathway followed by engagement of the alternative pathway amplification loop. The observation that complement deposition on viable AR spermatozoa was C1q dependent, but $\mathrm{Ab}$ independent, led us to investigate CRP as the activating pro-

\section{Figure 7}

Western blot of C3 fragments on AR spermatozoa. (A) Diagram of C3 structure. C3 is composed of 2 chains, $\alpha$ and $\beta$. Upon activation, C3a is released, leaving the truncated $\alpha^{\prime}$ chain of C3b. Factor I cleaves the $\alpha^{\prime}$ chain in 2 locations, creating the fragments $\alpha 1, \alpha 2$, and C3f (pictured in yellow). The interchain and intrachain disulfide bonds are also shown. (B) Spermatozoa were incubated with calcium ionophore to induce the acrosome reaction, exposed to $10 \%$ autologous human serum, lysed, absorbed with Sepharose linked to mAb against C3d, eluted in reducing SDS buffer, and loaded on a 10-20\% gel. Lanes 1-3, C3 standards; lane 4, AR spermatozoa incubated with 10\% serum; lane 5, spermatozoa were preincubated with the mAb TRA-2-10 and then exposed to $10 \%$ serum; lane 6 , spermatozoa were preincubated with the mAb GB24, which blocks the complement-regulatory function of MCP; lane 7, serum was preincubated with the $\mathrm{mAb} M H-10$, which blocks the complement-regulatory function of factor $\mathrm{H}(46)$.

tein, because this lectin initiates the complement cascade through C1q (42). Also, CRP concentrations in follicular fluid are comparable to those in serum and fluctuate with the female cycle, peaking during ovulation $(43,44)$. Further, since CRP activates complement on apoptotic cells $(42,47)$ and nonviable spermatozoa activate complement (48), we used 3 different nucleic dyes and examined motility to establish that viable, motile, AR spermatozoa activate complement. CRP bound specifically to the IAM of viable, AR spermatozoa. Consistent with its biochemical properties, heat inactivation or EDTA treatment of serum abrogated the binding of CRP to AR spermatozoa. In addition, serum incubated with phosphocholine beads to absorb CRP prevented C3d deposition. Taken together, these data indicate that CRP mediates the complement activation on the IAM of AR spermatozoa.

At first glance, complement activation on viable, AR spermatozoa is perplexing. If the membrane attack complex were to be formed on the IAM, there is the risk of membrane perturbation and possibly lysis of spermatozoa in the final steps of fertilization. Second, spermatozoa opsonized with C3b and C3bi are subject to immune adherence and phagocytosis. Third, the anaphylatoxins produced during complement activation could produce an inflammatory cell influx. Each of these points will now be addressed.

Surprisingly, complement activation on AR spermatozoa did not lead to loss of motility or cell death. These data suggest that the complement deposition on AR spermatozoa is largely restricted to early complement components (C1, C4, C2, and C3) and does not efficiently proceed to the membrane attack complex. The presence of 2 complement regulators on the IAM, MCP $(19,20,49)$ and DAF $(40)$, makes it difficult to envision spontaneous complement deposition on this membrane. However, activation by the classical pathway can overwhelm these regulatory proteins (46). Their presence, though, is in concert with the hypothesis of "restricted activation" (see below) and may account

A
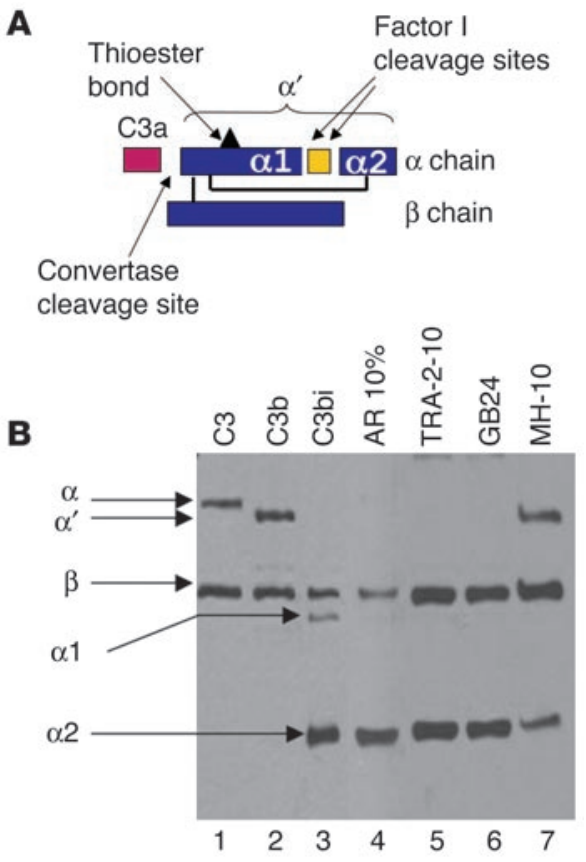
for why the late complement components are inefficiently deposited on viable, AR spermatozoa. These results are also consistent with studies showing that complement activation by CRP does not deplete the late complement components $(42,47)$.

Nonviable spermatozoa are known to activate the complement cascade, producing the anaphylatoxins C3a and C5a $(50,51)$. These peptides are potent chemotaxins and cellular activators that could serve to attract neutrophils to clear the female reproductive tract of nonviable spermatozoa $(50,51)$. It is also likely that these neutrophils assist in eliminating pathogens introduced to the female genital tract. In concert with this idea, the concentration of C3 in follicular fluid increases during ovulation (52). Under this scenario, C3b-opsonized viable spermatozoa might be at risk for phagocytosis. However, complement activation on AR spermatozoa occurs following binding to the zona pellucida of the egg. There is limited, if any, opportunity for these spermatozoa to interact with a phagocyte after entering the zona pellucida. As outlined above, complement activation on nonviable spermatozoa is likely a means of affecting their clearance. Viable AR spermatozoa, in contrast, induce restricted targeted complement activation for a different purpose, presumably to facilitate sperm-egg interactions.

The purpose of complement activation on AR spermatozoa may be to coat the IAM with opsonins. Complement fragments on spermatozoa would then facilitate egg binding, similar to complement's role in mediating immune adherence. Complement receptors 1 and 3 are expressed on the plasma membrane of the egg (29). Complement is visualized as part of a highly redundant process to facilitate sperm-egg binding. This line of reasoning could explain why the mice deficient in C3 $(53,54), \mathrm{C} 4$ (55), MCP (24), or integrins (56) are fertile. We hypothesize that dismantling parts of the complement system in these mice eliminates a supportive pathway for fertilization but does not obliterate it. Hence, by the broad measurement of litter size, there is no defect observed. This hypothesis provides the impetus to further assess the sperm-egg binding in these deficient mouse strains.

The possibility of complement activation fragments interacting with complement receptors to facilitate sperm-egg binding was first proposed in 1993 by Anderson and colleagues (29). In that pivotal report, several mechanisms were identified whereby spermatozoa could be coated with C3b, including alternative pathway activation, direct cleavage of $\mathrm{C} 3$ by the protease acrosin, and binding of dimeric $\mathrm{C} 3 \mathrm{~b}$ to complement regulators/receptors. We have extended and refined this study, particularly in relation to the complement activation on AR spermatozoa. In an experimental system that quantitatively assesses viability, acrosomal status, and complement deposition using follicular fluid, we demonstrate that CRP binds to the IAM of viable AR spermatozoa and initiates the classical pathway. These results are consistent with a "seeding" of $\mathrm{C} 3 \mathrm{~b}$ by the classical pathway that in turn triggers the feedback loop of the alternative pathway. The bound C3b is rapidly converted to C3bi by factors $\mathrm{H}$ and I. We therefore hypothesize that C3bi, a ligand for integrin-related complement receptors, facilitates sperm-egg interactions.

Complement activation on viable AR spermatozoa, whether spontaneous or induced by the calcium ionophore, has several features that distinguish it from complement activation on foreign antigens. First, on spermatozoa, the fragment deposition is targeted to a specific cellular location. Second, complement activation is restricted beyond the $\mathrm{C} 3$ step, thereby limiting the formation of membrane-damaging pores and release of the C5a chemotactic factor and anaphylaxation. Third, the overall magnitude of activation up to the C3 step appears to be more tightly controlled. It is as if this system on spermatozoa was specifically designed to allow a measured amount of $\mathrm{C} 3 \mathrm{~b}$ to be deposited at a specific site for a critical purpose. This concept of complement activation on self tissue (CAST), which we propose to also call TRACS for highly targeted and restricted activation of the complement system, is not well described as such in the literature; however, we believe it is a common and underappreciated function of the complement system's interaction with self tissue. In conditions like apoptosis, ischemia/reperfusion injury, and probably other types of alterations in host cell structure, TRACS is a preferable means to activate the complement system. In contrast to the desirable explosion of the cascade on microbes, complement activation on an apoptotic cell is less robust, yet sufficient to facilitate the safe and relatively noninflammatory removal of altered self $(42,47)$. In the setting of ischemia/reperfusion injury, one can envision a gradient of complement activation that correlates with the degree of cellular damage. In this example, the goal is to rapidly and safely remove dead and dying tissue in order to enhance wound healing and promote tissue sterility. As previously outlined, activation on spermatozoa may represent a situation whereby complement activation plays a role in reproduction. In these settings, regulatory proteins are present on the cells, and it is likely the ratio of the strength and type of activation to the quantity and efficiency of the regulators that determines the outcome. Complement activation on the membrane of Mycobacterium tuberculosis may represent an abuse of this type of a system (57). This pathogen salvages the catalytic complement fragment $\mathrm{C} 2 \mathrm{a}$ of the classical pathway $\mathrm{C} 3$ convertase and uses it to then deposit a limited amount of $\mathrm{C} 3 \mathrm{~b}$ on its membrane. This deposition of $\mathrm{C} 3 \mathrm{~b}$ aids the pathogen by facilitating internalization through complement receptors.

In these experiments, one question that we set out to address was the role of MCP on the IAM of spermatozoa. A possibility entertained was that MCP regulated complement on the IAM. However, blocking MCP with mAbs does not enhance or diminish C3 fragment deposition, and MCP is not responsible for the cleavage of $\mathrm{C} 3 \mathrm{~b}$ to $\mathrm{C} 3 \mathrm{bi}$. MCP efficiently regulates the $\mathrm{C} 5$ convertase $(47,58)$. We therefore favor the idea that, along with DAF, CD59, factor $\mathrm{H}$, and CRP, MCP limits complement activation, especially C5 convertase activity. However, blocking MCP function with $\mathrm{mAb}$ did not increase C5b-9 deposition. Activation of complement via CRP leads to restricted C5b-9 activation $(42,47)$, so MCP's role in this situation may be redundant. A second possible role is that MCP may mediate a direct binding event between the spermatozoa and the egg. MCP has been shown to associate with the tetraspan CD9 on macrophages (59) and other cell types (60). The targeted disruption of CD9 in mice results in infertility due to the inability of spermatozoa to bind to the egg $(61,62)$. Perhaps integrins associated with MCP, CD9, and tetraspans mediate binding between the spermatozoa and the egg. Alternatively, as previously suggested, MCP may be one part of a C3b dimer bridge with complement receptors on the egg (29), analogous to complement's role in mediating immune adherence. A third possibility is that complement deposition cross-links MCP on the IAM, inducing MCP-mediated signaling events important for fertilization (signaling through MCP is reviewed in ref. 13). A fourth possibility is that MCP influences membrane fluidity, as 
evidenced by a role for MCP in the acceleration of the rate of the acrosome reaction in MCP-deficient mice (24).

In summary, viable AR spermatozoa activate complement. The complement deposition is triggered by CRP activation of the classical pathway, followed by amplification of C3 deposition through the alternative pathway feedback loop. Complement deposition is localized to the IAM and is curtailed beyond the C3 activation step. The purpose of this reaction remains problematic. However, we propose that this type of targeted and restricted complement activation is a common phenomenon on modified self tissue.

\section{Methods}

Spermatozoa samples. Spermatozoa samples were obtained with informed consent, and all studies were approved by the Washington University Human Studies Committee (protocol number 94-0420). Human spermatozoa samples were repeatedly obtained from 4 healthy donors ranging in age from 18 to 38 . Fresh ejaculates were allowed to liquefy at room temperature for 45 minutes, and then washed twice with modified human tubal fluid (mHTF) supplemented with $5 \%$ serum substitute supplement (Irvine Scientific) and 5\% HSA (100 mg/ml) (Irvine Scientific). Unless stated otherwise, centrifugation steps were performed at $200 \mathrm{~g}$ for 10 minutes at $4^{\circ} \mathrm{C}$.

Serum preparation. Blood samples were obtained from donors that provided informed consent, and all studies were approved by the Washington University Human Studies Committee (protocol number 94-0420). Human blood was collected in glass tubes and allowed to clot at room temperature for 30 minutes. Samples were then centrifuged at $200 \mathrm{~g}$. The serum was placed in sterile $1.5-\mathrm{ml}$ microcentrifuge tubes and maintained at $-20^{\circ} \mathrm{C}$ for short-term or $-70^{\circ} \mathrm{C}$ for long-term storage.

Follicular fluid collection. Follicular fluid samples were obtained from patients that provided informed consent, and all studies were approved by the Washington University Human Studies Committee (protocol number 02-1004). Follicular fluids were collected from patients undergoing in vitro fertilization. Ovarian stimulation was performed using gonadotropins in combination with a gonadotropin-releasing hormone (GnRH) antagonist or GnRH agonist according to established protocols. Serum estradiol was measured on cycle days 3, 5, and 7 and daily thereafter until retrieval. Vaginal ultrasound was performed daily from cycle day 7 onward. Human chorionic gonadotropin (5,000-10,000 IU, Profasi; Serono Inc.) was administered intramuscularly when 3 or more follicles were at least $16 \mathrm{~mm}$ in largest diameter. Transvaginal follicular aspiration was performed 35-36 hours later. Follicles were aspirated using a Wallace catheter (Irvine Scientific), and contents of each follicle were collected into a 15-ml tube (Falcon 2057; Cardinal Health) containing approximately $1.0 \mathrm{ml}$ of $\mathrm{mHTF}$ (Irvine Scientific) supplemented with $0.3 \mathrm{ml}$ of sodium heparin. After the removal of oocytes, the follicular fluids were stored at $4{ }^{\circ} \mathrm{C}$ until processed. To collect pure follicular fluid, the dominant follicle of a patient(s) was aspirated directly into a tube that did not contain mHTF.

Acrosome reaction. The acrosome reaction was induced by incubation of spermatozoa in supplemented mHTF media with $20 \mathrm{mM}$ calcium ionophore $\mathrm{A} 23187$ (Sigma-Aldrich) at $37^{\circ} \mathrm{C}$ for 45 minutes.

Antibodies. The mouse anti-MCP mAb TRA-2-10 was prepared at Washington University. The mouse $\mathrm{mAb}$ against factor $\mathrm{H}(\mathrm{MH}-10)$ was donated by J. Lambris (University of Pennsylvania, Philadelphia, Pennsylvania, USA). The mouse mAbs against human C $3 \mathrm{~d}$ and $\mathrm{C} 5 \mathrm{~b}-9$ neoantigen were purchased from Quidel Corp. The polyclonal goat anti-human C9 Ab was purchased from Quidel Corp. The polyclonal goat anti-human C5 $\mathrm{Ab}$ was purchased from Advanced Research Technologies. Polyclonal goat anti-human CRP was purchased from Affinity BioReagents. HRPconjugated rabbit anti-goat IgG (Sigma-Aldrich) was used as the secondary $\mathrm{Ab}$ for Western blotting. Zenon labeling kits (Invitrogen Corp.) were used to link fluorochromes (Alexa Fluor 488 or Alexa Fluor 647) to primary Abs according to the manufacturer's instructions ( $1 \mu \mathrm{g}$ of primary $\mathrm{Ab}$ was used per labeling reaction). One microgram of TRA-2-10 was used per $1 \times 10^{6}$ spermatozoa, and $1 \mu \mathrm{g}$ of either the C $3 \mathrm{~d}$ or the C5b-9 $\mathrm{mAb}$ was used per $2 \times 10^{6}$ spermatozoa for both FACS (BD Biosciences) and confocal microscopy.

Complement deposition. AR or untreated spermatozoa were resuspended in gelatin veronal buffer supplemented with $\mathrm{Mg}^{2+}$ and $\mathrm{Ca}^{2+}\left(\mathrm{GVB}^{++}\right)$(SigmaAldrich). After incubation in $10 \%$ autologous serum or follicular fluid for 15 minutes at $37^{\circ} \mathrm{C}$, the samples were washed with supplemented $\mathrm{mHTF}$ media. Gelatin veronal buffer supplemented with 10 mM EDTA (SigmaAldrich) and gelatin veronal buffer supplemented with 10 mM EGTA and $7 \mathrm{mM}$ magnesium chloride were also used. Sera were heat-inactivated by 30 minutes of incubation at $56^{\circ} \mathrm{C}$. Sources of the $\mathrm{C} 2$ - and C7-deficient sera were as described in ref. $46 . \mathrm{C} 1 \mathrm{q}$ - and factor B-depleted sera were obtained from Advanced Research Technologies.

Viability dyes. Propidium iodide (Invitrogen Corp.), SYTOX Green (Invitrogen Corp.), and SYBR 14 (Invitrogen Corp.) were used to assess viability according to the manufacturer's instructions. Results were comparable with all 3 dyes, but the different fluorescent ranges of the 3 dyes allowed for flexibility in fluorochrome usage.

FACS analysis. Spermatozoa, after exposure to a complement source, were incubated with Zenon-labeled primary Abs (described above) for 30 minutes at $37^{\circ} \mathrm{C}$ in supplemented mHTF and then washed twice. Flow cytometry was performed with a FACSCalibur (BD Biosciences) using forward and side scatter thresholds optimized for spermatozoa. A minimum of 10,000 events were collected per sample. Samples were gated on viable spermatozoa.

Lysates. Spermatozoa were resuspended in lysis buffer (PBS containing $1 \%$ NP-40, $0.05 \%$ SDS, and Complete protease inhibitor cocktail tablets [Roche Diagnostics Corp.]) and incubated for 30 minutes at $4^{\circ} \mathrm{C}$. Samples were centrifuged at $13,500 \mathrm{~g}$ for 15 minutes at $4^{\circ} \mathrm{C}$. Supernatants were transferred to $1.5-\mathrm{ml}$ microcentrifuge tubes and stored at $-20^{\circ} \mathrm{C}$.

Immunoabsorption. Polyclonal goat anti-human $\mathrm{C} 3$ was coupled to $\mathrm{CNBr}-$ activated Sepharose (Amersham Biosciences) according to the manufacturer's suggestion. Spermatozoa lysate $(50 \mu \mathrm{l})$, coupled Sepharose $(50 \mu \mathrm{l})$, protease inhibitor tablets (Roche Diagnostics Corp.), and cell lysis buffer were combined and incubated at $4{ }^{\circ} \mathrm{C}$ overnight with "end over end" mixing. The Sepharose beads were next washed 3 times with $0.3 \%$ NP-40. Eluates, obtained by boiling of the Sepharose beads in SDS loading buffer, were used for Western blotting as described below.

Western blotting. Cell lysates were separated in a 10-20\% SDS-polyacrylamide gel under reducing conditions and then transferred onto nitrocellulose membranes using the NOVEX MiniCell II wet blotting system (Invitrogen Corp.). The membranes were blocked with $5 \%$ nonfat dry milk powder in $1 \times$ Tris-buffered saline (TBS) and 0.05\% Tween-20 (TBS-T) (Sigma-Aldrich) overnight at $4^{\circ} \mathrm{C}$. After 3 washes in TBS-T, the membranes were incubated in blocking buffer for 1 hour at $37^{\circ} \mathrm{C}$ with either purified polyclonal goat anti-CRP $(1: 1,000)$ or polyclonal goat anti-C3 $\mathrm{Ab}(1: 7,500)$. Blots were washed 3 times and then incubated in blocking buffer for 1 hour at room temperature with HRP-conjugated rabbit anti-goat IgG secondary Ab (Sigma-Aldrich) (1:10,000). After washing, the signals were developed using the enhanced chemiluminescence kit from Pierce Biotechnology Inc. The bands were visualized by exposure of the membranes to BioMax MR film (Eastman Kodak Co.).

ELISA for buman IgM. The Microwell ELISA (Diagnostic Automation Inc.) was used to assess total human IgM in spermatozoa lysates according to the manufacturer's instructions. 
Confocal microscopy. Spermatozoa were incubated in the fluid phase with Zenon-labeled primary Abs (described above) for 30 minutes at $37^{\circ} \mathrm{C}$ in supplemented mHTF. After the samples were washed twice with supplemented mHTF media, a 1:1 dilution of sample and ProLong Antifade Reagent (Invitrogen Corp.) was placed on a slide and analyzed with an LSM 510 META confocal microscope (Carl Zeiss Inc.).

CRP absorption. Immobilized $p$-aminophenyl phosphorylcholine beads (150 $\mu \mathrm{l}$; Pierce Biotechnology Inc.) were added to human serum diluted $1: 10$ with $\mathrm{GVB}^{++}$buffer. The samples were incubated at room temperature for 30 minutes with gentle mixing. After centrifugation at $200 \mathrm{~g}$ for 5 minutes, the supernatant was removed and used as a complement source for FACS experiments.

\section{Acknowledgments}

We thank Madonna Bogacki for excellent secretarial support and Wandy Beaty from the Washington University Imaging Core for guidance in confocal microscopy. This work was supported by NIH grant AI37618-10.

Received for publication August 30, 2004, and accepted in revised form March 1, 2005.

Address correspondence to: John P. Atkinson, Washington University School of Medicine, 660 South Euclid Avenue, Campus Box 8045, St. Louis, Missouri 63110, USA. Phone: (314) 362-8391; Fax: (314) 362-1366; E-mail: jatkinso@im.wustl.edu.
1. Seya, T., Turner, J., and Atkinson, J.P. 1986. Purification and characterization of a membrane protein (gp45-70) that is a cofactor for cleavage of C3b and C4b. J. Exp. Med. 163:837-855.

2. Ballard, L.L., Bora, N.S., Yu, G.H., and Atkinson, J.P. 1988. Biochemical characterization of membrane cofactor protein of the complement system. J. Immunol. 141:3923-3929.

3. Seya, T., and Atkinson, J.P. 1989. Functional properties of membrane cofactor protein of complement. Biochem. J. 264:581-588.

4. Oglesby, T.J., Allen, C.J., Liszewski, M.K., White, D.J., and Atkinson, J.P. 1992. Membrane cofactor protein (CD46) protects cells from complementmediated attack by an intrinsic mechanism. J. Exp. Med. 175:1547-1551.

5. Dorig, R.E., Marcil, A., Chopra, A., and Richardson, C.D. 1993. The human CD46 molecule is a receptor for measles virus (Edmonston strain). Cell. 75:295-305.

6. Naniche, D., et al. 1993. Human membrane cofactor protein (CD46) acts as a cellular receptor for measles virus. J. Virol. 67:6025-6032.

7. Manchester, M., Liszewski, M.K., Atkinson, J.P., and Oldstone, M.B.A. 1994. Multiple isoforms of CD46 (membrane cofactor protein) serve as receptors for measles virus. Proc. Natl. Acad. Sci. U. S. A. 91:2161-2165.

8. Santoro, F., et al. 1999. CD46 is a cellular receptor for human herpesvirus 6. Cell. 99:817-827.

9. Segerman, A., et al. 2003. Adenovirus type 11 uses CD46 as a cellular receptor. J. Virol. 77:9183-9191.

10. Gaggar, A., Shayakhmetov, D.M., and Lieber, A. 2003. CD46 is a cellular receptor for group B adenoviruses. Nat. Med. 9:1408-1412.

11. Okada, N., Liszewski, M.K., Atkinson, J.P., and Caparon, M. 1995. Membrane cofactor protein (CD46) is a keratinocyte receptor for the M protein of the group A streptococcus. Proc. Natl. Acad. Sci. U. S. A. 92:2489-2493.

12. Kallstrom, H., Liszewski, M.K., Atkinson, J.P., and Jonsson, A.B. 1997. Membrane cofactor protein (MCP or CD46) is a cellular pilus receptor for pathogenic Neisseria. Mol. Microbiol. 25:639-647.

13. Riley-Vargas, R.C., and Atkinson, J.P. 2003. Expression of membrane cofactor protein (MCP; CD46) on spermatozoa: just a complement inhibitor? Modern Aspects of Immunobiology. 3:75-78.

14. Kemper, C., et al. 2003. Activation of human CD4+ cells with CD3 and CD46 induces a T-regulatory cell 1 phenotype. Nature. 421:388-392.

15. Richards, A., et al. 2003. Mutations in human complement regulator, membrane cofactor protein (CD46), predispose to development of familial hemolytic uremic syndrome. Proc. Natl. Acad. Sci. U. S. A. 100:12966-12971.

16. Noris, M., et al. 2003. Familial haemolytic uraemic syndrome and an MCP mutation. Lancet. 362:1542-1547.

17. Cervoni, F., et al. 1992. Identification and charac- terization of membrane cofactor protein (MCP) on human spermatozoa. J. Immunol. 148:1431-1437.

18. Riley, R.C., Kemper, C., Leung, M., and Atkinson, J.P. 2002. Characterization of human membrane cofactor protein (MCP; CD46) on spermatozoa. Mol. Reprod. Dev. 62:534-546.

19. Anderson, D.J., Michaelson, J.S., and Johnson, P.M. 1989. Trophoblast/leukocyte-common antigen is expressed by human testicular germ cells and appears on the surface of acrosome-reacted sperm. Biol. Reprod. 41:285-293.

20. Fenichel, P., et al. 1990. Localization and characterization of the acrosomal antigen recognized by GB24 on human spermatozoa. Mol. Reprod. Dev. 27:173-178.

21. Kitamura, M., et al. 1997. Possible association of infertility with sperm-specific abnormality of CD46. J. Reprod. Immunol. 33:83-88.

22. Nomura, M., et al. 2001. Genomic analysis of idiopathic infertile patients with sperm-specific depletion of CD46. Exp. Clin. Immunogenet. 18: $42-50$.

23. Jiang, H., and Pillai, S. 1998. Complement regulatory proteins on the sperm surface: relevance to sperm motility. Am. J. Reprod. Immunol. 39:243-248.

24. Inoue, N., et al. 2003. Disruption of mouse CD46 causes an accelerated spontaneous acrosome reaction in sperm. Mol. Cell. Biol. 23:2614-2622.

25. Mizuno, M., Harris, C.L., and Morgan, B.P. 2004 Immunohistochemical analysis of membrane complement regulatory proteins in rat testis: unique roles for DAF and MCP in spermatozoal function? Abstracts from the XXth International Complement Workshop, 172. Mol. Immunol. 41:280.

26. Hosokawa, M., Nonaka, M., Okada, N., and Okada, H. 1996. Molecular cloning of guinea pig membrane cofactor protein: preferential expression in testis. J. Immunol. 157:4946-4952.

27. Foley, S., Li, B., Dehoff, M., Molina, M., and Holers, V.M. 1993. Mouse Crry/p65 is a regulator of the alternative pathway of complement activation. Eur. J. Immunol. 23:1381-1384.

28. Xu, C., et al. 2000. A critical role for the murine complement regulator Crry in fetomaternal tolerance. Science. 287:498-501.

29. Anderson, D.J., Abbott, A.F., and Jack, R.M. 1993. The role of complement component $\mathrm{C} 3 \mathrm{~b}$ and its receptors in sperm-oocyte interaction. Proc. Natl. Acad. Sci. U. S. A. 90:10051-10055.

30. Taylor, C.T., Biljan, M.M., Kingsland, C.R., and Johnson, P.M. 1994. Inhibition of human spermatozoon-oocyte interaction in vitro by monoclonal antibodies to CD46 (membrane cofactor protein). Hum. Reprod. 9:907-911.

31. D’Cruz, O.J., Lambert, H., and Haas, G.G., Jr. 1997. Expression of CD15 (Lewisx) antigen on human sperm and its role in sperm-egg interaction. Am.J. Reprod. Immunol. 37:172-183.

32. Riley, R.C., Tannenbaum, P.L., Abbott, D.H., and Atkinson, J.P. 2002. Cutting edge. Inhibiting mea- sles virus infection but promoting reproduction: an explanation for splicing and tissue-specific expression of CD46. J. Immunol. 169:5405-5409.

33. Hsu, E.C., et al. 1997. Artificial mutations and natural variations in the CD46 molecules from human and monkey cells define regions important for measles virus binding. J. Virol. 71:6144-6154.

34. Adams, E., Brown, M.C., Nunge, M., Krych, M., and Atkinson, J.P. 1991. Contribution of the repeating domains of membrane cofactor protein (CD46) of the complement system to ligand binding and cofactor activity. J. Immunol. 147:3005-3011.

35. Iwata, K., et al. 1995. Diversity of sites for measles virus binding and for inactivation of complement $\mathrm{C} 3 \mathrm{~b}$ and $\mathrm{C} 4 \mathrm{~b}$ on membrane cofactor protein CD46. J. Biol. Chem. 270:15148-15152.

36. Vanderpuye, O.A., Labarrere, C.A., and McIntyre, J.A. 1992. The complement system in human reproduction. Am. J. Reprod. Immunol. 27:145-155.

37. Clarke, G.N., Hsieh, C., Koh, S.H., and Cauchi, M.N. 1984. Sperm antibodies, immunoglobulins, and complement in human follicular fluid. Am.J. Reprod. Immunol. 5:179-181.

38. Perricone, R., et al. 1992. Functionally active complement is present in human ovarian follicular fluid and can be activated by seminal plasma. Clin. Exp. Immunol. 89:154-157.

39. Perricone, R., et al. 1990. Complement, complement activation and anaphylatoxins in human ovarian follicular fluid. Clin. Exp. Immunol. 82:359-362.

40. Cervoni, F., et al. 1993. Expression of decay accelerating factor (CD55) of the complement system on human spermatozoa. J. Immunol. 151:939-948.

41. Fenichel, P., et al. 1994. Expression of the complement regulatory protein CD59 on human spermatozoa: characterization and role in gametic interaction. Mol. Reprod. Dev. 38:338-346.

42. Mold, C., Gewurz, H., and Du Clos, T.W. 1999. Regulation of complement activation by C-reactive protein. Immunopharmacology. 42:23-30.

43. Jilma, B., et al. 1997. Menstrual cycle-associated changes in blood levels of interleukin- 6 , alpha1 acid glycoprotein, and C-reactive protein. J. Lab. Clin. Med. 130:69-75.

44. Orvieto, R., et al. 2004. C-reactive protein levels in patients undergoing controlled ovarian hyperstimulation for IVF cycle. Hum. Reprod. 19:357-359.

45. Liszweski, M.K., et al. 2000. Dissecting sites important for complement regulatory activity in membrane cofactor protein (MCP; CD46). J. Biol. Chem. 275:37692-37701.

46. Barilla-LaBarca, M.L., Liszewski, M.K., Lambris, J.D., Hourcade, D., and Atkinson, J.P. 2002. Role of membrane cofactor protein (CD46) in regulation of $\mathrm{C} 4 \mathrm{~b}$ and $\mathrm{C} 3 \mathrm{~b}$ deposited on cells. J. Immunol. 168:6298-6304.

47. Gershov, D., Kim, S., Brot, N., and Elkon, K.B. 2000. C-Reactive protein binds to apoptotic cells, protects the cells from assembly of the 
terminal complement components, and sustains an antiinflammatory innate immune response: implications for systemic autoimmunity [erratum 2001, 193:1439]. J. Exp. Med. 192:1353-1364.

48. Vogelpoel, F.R., et al. 1987. Antibody and complement-binding activity of viable and nonviable human spermatozoa. Arch. Androl. 18:189-197.

49. Cervoni, F., et al. 1992. Identification and characterization of membrane cofactor protein on human spermatozoa. J. Immunol. 148:1431-1437.

50. Clark, R.A., and Klebanoff, S.J. 1976. Generation of a neutrophil chemotactic agent by spermatozoa: role of complement and regulation by seminal plasma factors. J. Immunol. 117:1378-1386.

51. Vogelpoel, F.R., and Verhoef, J. 1985. Activation of polymorphonuclear leukocytes by spermatozoa. Arch. Androl. 14:123-131.

52. Lee, Y.-L., et al. 2004. The embryotrophic activity of oviductal cell-derived complement $\mathrm{C} 3 \mathrm{~b}$ and iC $3 b$, a novel function of complement protein in reproduction. J. Biol. Chem. 279:12763-12768.

53. Wessels, M.R., et al. 1995. Studies of group B streptococcal infection in mice deficient in complement component $\mathrm{C} 3$ or C4 demonstrate an essential role for complement in both innate and acquired immunity. Proc. Natl. Acad. Sci. U. S. A. 92:11490-11494.

54. Circolo, A., et al. 1999. Genetic disruption of the murine complement $\mathrm{C} 3$ promoter region generates deficient mice with extrahepatic expression of C3 mRNA. Immunopharmacology. 42:135-149.

55. Fischer, M., et al. 1996. Regulation of the B cell response to $\mathrm{T}$-dependent antigens by classical pathway complement. J. Immunol. 157:549-556.

56. He, Z.Y., et al. 2003. None of the integrins known to be present on the mouse egg or to be ADAM receptors are essential for sperm-egg binding and fusion. Dev. Biol. 254:226-237.

57. Schorey, J.S., Carroll, M.C., and Brown, E.J. 1997. A macrophage invasion mechanism of pathogenic mycobacteria. Science. 277:1091-1093.

58. Seya, T., et al. 1991. Preferential inactivation of the C5 convertase of the alternative complement pathway by factor I and membrane cofactor protein (MCP). Mol. Immunol. 28:1137-1147.

59. Kurita-Taniguchi, M., et al. 2002. Molecular assembly of CD46 with CD9, alpha 3-beta 1 integrin and protein tyrosine phosphatase SHP-1 in human macrophages through differentiation by GM-CSF. Mol. Immunol. 38:689-700.

60. Lozahic, S., et al. 2000. CD46 (membrane cofactor protein) associates with multiple beta 1 integrins and tetraspans. Eur. J. Immunol. 30:900-907.

61. LeNaour, F., Rubinstein, E., Jasmin, C., Prenant, M., and Boucheix, C. 2000. Severely reduced female fertility in CD9-deficient mice. Science. 287:319-321.

62. Miyado, K., et al. 2000. Requirement of CD9 on the egg plasma membrane for fertilization. Science. 287:321-324. 